\title{
Definably compact abelian groups
}

\author{
Mário J. Edmundo* \\ CMAF Universidade de Lisboa \\ Av. Prof. Gama Pinto 2 \\ 1649-003 Lisboa, Portugal \\ edmundo@cii.fc.ul.pt
}

\author{
Margarita Otero ${ }^{\dagger}$ \\ Departamento de Matemáticas \\ Universidad Autónoma de Madrid \\ 28049 Madrid, Spain \\ margarita.otero@uam.es
}

\begin{abstract}
Let $\mathbf{M}$ be an o-minimal expansion of a real closed field. Let $G$ be a definably compact definably connected abelian $n$-dimensional group definable in $\mathbf{M}$. We show the following: the o-minimal fundamental group of $G$ is isomorphic to $\mathbb{Z}^{n}$; for each $k>0$, the $k$-torsion subgroup of $G$ is isomorphic to $(\mathbb{Z} / k \mathbb{Z})^{n}$, and the o-minimal cohomology algebra over $\mathbb{Q}$ of $G$ is isomorphic to the exterior algebra over $\mathbb{Q}$ with $n$ generators of degree one.
\end{abstract}

\section{Introduction}

We work over an o-minimal expansion $\mathbf{M}$ of a real closed field, and by definable we mean definable (with parameters) in $\mathbf{M}$. As it is known $\mathrm{o}^{-}$minimal structures are generalitations of semialgebraic and subanalytic structures. We are specially interested in definable groups, that is, groups for which both the set and the graph of the group operation are definable. The starting point in the study of definable groups is the work of Pillay in [11], where he proved that such a group is a topological group equipped with a definable manifold structure. Because we are working over an expansion of a real closed field we can suppose that the topology in the group is induced by that of the ambient space (see Robson's Theorem in van den Dries [6] p.159, noting that a topological group is a regular space), and so in this paper by definable manifold we mean an affine definable manifold. Peterzil and

\footnotetext{
*Supported by the FCT grant SFRH/BPD/6015/2001 and partially by the European Research and Training Network RAAG HPRN-CT-2001-00271

${ }^{\dagger}$ Partially supported by BFM-2002-04797 and the European Research and Training Network RAAG HPRN-CT-2001-00271
} 
Steinhorn introduce in [10] the concept of definably compact (o-minimal analogue to the semialgebraic complete, see Delfs and Knebusch [4]), and they prove that definably compact is equivalent to closed and bounded, when the topology is induced by that of the ambient space. The main result of this paper concerns definably compact definably connected abelian definable groups. By the results in [11] definable groups resemble real Lie groups, so the definable groups we are concern with should resemble tori. However in [10] an example is given of such a group without infinite definable proper subgroups. Actually, in the o-minimal context, even in the semialgebraic one, we do not have a classification theorem of definably compact definably connected abelian definable groups as we have in the Lie case (see Peterzil and Starchenko [9] for more results on abelian definable groups). Here we show that such a definable group has the o-minimal topology of a torus in the following sense:

Theorem 1.1 (Structure Theorem) Let G be a definably compact definably connected abelian definable group of dimension $n$. Then,

(a) the o-minimal fundamental group of $G$ is isomorphic to $\mathbb{Z}^{n}$;

(b) for each $k>0$, the $k$-torsion subgroup of $G$ is isomorphic to $(\mathbb{Z} / k \mathbb{Z})^{n}$, and

(c) the o-minimal cohomology algebra over $\mathbb{Q}$ of $G$ is isomorphic to the exterior algebra over $\mathbb{Q}$ with $n$ generators of degree one.

For the definition of o-minimal fundamental group see Section 2. There, we also introduce definable covering maps and their groups of definable covering transformations and prove their basic properties. We then apply these results to definable groups getting a sufficient condition for a map to be a definable covering map (Proposition 2.11). We end the section by proving, for definably connected abelian definable groups, the existence of some $s \geq 0$ satifying (a) and (b) of the Structure Theorem with $s$ instead of $n$ (Theorem 2.1).

In Section 3 we first consider o-minimal homology studied by Woerheide in [13] (see also Edmundo and Woerheide [7]), and we adapt a proof of the Eilenberg-Zilber Theorem to the o-minimal context (Proposition 3.2). After defining o-minimal cohomology with coefficients in $\mathbb{Q}$ we can then prove an o-minimal version of the Künneth Formula for cohomology (Corollary 3.3). With these tools at hand we can follow the classical proofs and equip the cohomology graded $\mathbb{Q}$-vector space of a definable set with a structure of graded $\mathbb{Q}$-algebra in a functorial way (Theorem 3.4). When our definable set is a definably connected definable group the o-minimal cohomology 
$\mathbb{Q}$-algebra becomes, in analogy with the Lie case, a free commutative algebra over $\mathbb{Q}$ with odd degree generators, i.e., an exterior algebra over $\mathbb{Q}$ (Corollaries 3.5 and 3.6).

In Section 4 we consider definable manifolds and use the existence of the o-minimal fundamental class of an orientable definable manifold, proved by Beraducci and Otero in [2], to define the degree of a definable map between oriented definable manifolds of the same dimension and with the target manifold definably compact. We then prove properties of degree of the relevant maps which will be specially useful later for the definable groups we are interested on (Proposition 4.6).

Finally in Section 5 we first prove an o-minimal version of Poincaré-Hurewitz Theorem (Theorem 5.1) and then we apply the previous results to prove the Structure Theorem.

Implicitly, we work over the o-minimal site, which is the analogue of the semialgebraic site (see [4]), but we shall not mention it. For definitions and basic results on o-minimal structures we refer to [6]. To simplify notation we have include superscript def only in the definitions of the definable category, the philosophy is that each functor applies to the corresponding category. Some of the proofs are easy adaptations of classical proofs to the definable category, even if we state so in each relevant case, we have tried to include most of them to make the paper more readable.

\section{Definable covering maps}

Given a definable set $X$ and $x_{0} \in X$, the o-minimal fundamental group $\pi^{\operatorname{def}}\left(X, x_{0}\right)$ is defined in the usual way except that we use definable paths and definable homotopies. Berarducci and Otero prove in [1] that $\pi^{\operatorname{def}}\left(X, x_{0}\right)$ is a finitely presented group. If $f: X \rightarrow Y$ is a definable continuous map between definable sets with $f\left(x_{0}\right)=y_{0}$ then the induced map $f^{\pi}: \pi^{\text {def }}\left(X, x_{0}\right) \rightarrow$ $\pi^{\operatorname{def}}\left(Y, y_{0}\right):[\sigma] \mapsto f^{\pi}([\sigma])=[f \sigma]$ is a group homomorphism and we have the usual functorial properties (see definition 2.1 in [1]). We note that if $X$ is definably connected then $\pi^{\operatorname{def}}\left(X, x_{0}\right) \cong \pi^{\operatorname{def}}\left(X, x_{1}\right)$ for any $x_{0}, x_{1} \in X$, in this case we write $\pi^{\operatorname{def}}(X)$ instead of $\pi^{\operatorname{def}}\left(X, x_{0}\right)$. Note also that the o-minimal fundamental group is invariant under elementary extensions (see proofs of Theorem 1.1 and Corollary in [1]).

We shall omit the superscript "def".

The aim of this section is to introduce the notion of definable covering map and prove the following. 
Theorem 2.1 Let $G$ be a definably connected abelian definable group. Then there is an $s \in \mathbb{N}$ such that

(a) the o-minimal fundamental group $\pi(G)$ of $G$ is isomorphic to $\mathbb{Z}^{s}$, and

(b) the $k$-torsion subgroup of $G$ is isomorphic to $(\mathbb{Z} / k \mathbb{Z})^{s}$, for each $k>0$.

We begin with three lemmas which follow from the definition of o-minimal fundamental group exactly as in the classical case.

Lemma 2.2 Let $X$ and $Y$ be definable sets and let $x_{0} \in X$ and $y_{0} \in Y$. Then, $\theta: \pi\left(X, x_{0}\right) \times \pi\left(Y ; y_{0}\right) \rightarrow \pi\left(X \times Y,\left(x_{0}, y_{0}\right)\right):([\gamma],[\delta]) \mapsto \theta([\gamma],[\delta])=$ $[(\gamma, \delta)]$ is a group isomorphism.

Proof. The inverse of $\theta$ is $\left(q_{1}^{\pi}, q_{2}^{\pi}\right):[\rho] \mapsto\left(q_{1}^{\pi}[\rho], q_{2}^{\pi}[\rho]\right)$, where $q_{1}$ and $q_{2}$ are the projections onto $X$ and $Y$, respectively.

Lemma 2.3 Let $G$ be a definable group and let 1 denote its neutral element. Then $\pi(G, 1)$ is an abelian group.

Proof. Let $k_{1}$ be the constant loop at 1 , and let $e=\left[k_{1}\right]$. Let $m: G \times$ $G \rightarrow G$ be the multiplication in $G$. By definition of $\theta$ (above), we have $[\gamma]=m^{\pi} \theta(e,[\gamma])=m^{\pi} \theta([\gamma], e)$, for every $[\gamma] \in \pi(G, 1)$. Hence, for any $[\gamma],[\delta] \in \pi(G, 1),[\gamma] \cdot[\delta]=m^{\pi} \theta([\gamma], e) \cdot m^{\pi} \theta(e,[\delta])=m^{\pi} \theta(([\gamma], e) \cdot(e,[\delta]))=$ $m^{\pi} \theta([\gamma],[\delta])=m^{\pi} \theta((e,[\delta]) \cdot([\gamma], e))=[\delta] \cdot[\gamma]$.

Lemma 2.4 Let $G$ be a definable group. For each $k>0$, consider the continuous definable map $p_{k}: G \rightarrow G: a \mapsto a^{k}$, for each $a \in G$. Then, $\left(p_{k}\right)^{\pi}: \pi(G, 1) \rightarrow \pi(G, 1)$ takes $[\gamma]$ to $[\gamma]^{k}$, for each $[\gamma] \in \pi(G, 1)$.

Proof. By induction over $k$. Let $m$ denote the multiplication in $G$. Then $\left(p_{k+1}\right)^{\pi}([\gamma])=\left(m \circ\left(i d_{G}, p_{k}\right)\right)^{\pi}([\gamma])=m^{\pi}\left(\left[\left(\gamma, p_{k} \circ \gamma\right)\right]\right)$. By Lemma 2.2 and induction hypothesis, $m^{\pi}\left(\left[\left(\gamma, p_{k} \circ \gamma\right)\right]\right)=m^{\pi} \theta\left([\gamma],[\gamma]^{k}\right)$. By the proof of Lemma $2.3, m^{\pi} \theta\left([\gamma],[\gamma]^{k}\right)=[\gamma] \cdot[\gamma]^{k}=[\gamma]^{k+1}$.

The next step is to adapt the concept of covering map to the definable context and prove both the path lifting and the homotopy lifting properties of covering maps in the o-minimal context. The classical proofs of these properties cannot be applied because they make use of the Lebesgue number which it is not available in the $\mathrm{o}-$ minimal setting.

A definably continuous map $p: Y \rightarrow X$ between definably connected definable sets is a definable covering map if $p$ is onto and there is a finite family 
$\left\{U_{l}: l \in L\right\}$ of definably connected open definable subsets of $X$ such that $X=\bigcup_{l \in L} U_{l}$, and for each $l \in L$ and for each definably connected component $V$ of $p^{-1}\left(U_{l}\right)$, the map $p_{\mid V}: V \rightarrow U_{l}$ is a definable homeomorphism.

Let $p: Y \rightarrow X$ be a definable covering map and let $Z$ be a definable set and $f: Z \rightarrow X$ a definable continuous map. A definable continuous map $\tilde{f}: Z \rightarrow Y$ satisfying $p \tilde{f}=f$ is called a lifting of $f$. Note that both properties are preserved under elementary extensions.

As in the classical case we obtain the following useful lemma.

Lemma 2.5 (Unicity of liftings) Let $p: Y \rightarrow X$ be a definable covering map. Let $Z$ be a definably connected definable set and let $f: Z \rightarrow X$ be a definable continuous map. If $\tilde{f}_{1}$ and $\tilde{f}_{2}$ are two liftings of $f$, then $\tilde{f}_{1}=\tilde{f}_{2}$ provided there is a $z \in Z$ such that $\tilde{f}_{1}(z)=\tilde{f}_{2}(z)$.

Proof. Both sets $\left\{w \in Z \mid \tilde{f}_{1}(w)=\tilde{f}_{2}(w)\right\}$ and $\left\{w \in Z \mid \tilde{f}_{1}(w) \neq \tilde{f}_{2}(w)\right\}$ are definable and open, the first one is not empty.

Proposition 2.6 (Path Lifting) Let $p: Y \rightarrow X$ be a definable covering map. Let $\gamma$ be a definable path in $X$ and let $y \in Y$. If $p(y)=\gamma(0)$, then there is a unique definable path $\tilde{\gamma}$ in $Y$, lifting $\gamma$ and satisfying $\tilde{\gamma}(0)=y$. (The end point $\tilde{\gamma}(1)$ of such $\tilde{\gamma}$ will be denoted by $y * \gamma$.)

Proof. Let $X=\bigcup_{l \in L} U_{l}$, as in the definition of definable covering map. Then $[0,1]=\bigcup_{l \in L} \gamma^{-1}\left(U_{l}\right)$, with the $\gamma^{-1}\left(U_{l}\right)$ 's open in $[0,1]$. Then, by Lemma 6.3.6 in [6], for each $l \in L$ there is a $W_{l} \subset[0,1]$, open in $[0,1]$ such that $W_{l} \subset \overline{W_{l}} \subset \gamma^{-1}\left(U_{l}\right)$ and $[0,1]=\bigcup_{l \in L} W_{l}$. Therefore, there are $0=s_{0}<s_{1}<\cdots<s_{r}=1$ such that for each $i=0, \ldots, r-1$ we have $\gamma\left(\left[s_{i}, s_{i+1}\right]\right) \subset U_{l(i)}$ (and $\left.\gamma\left(s_{i+1}\right) \in U_{l(i)} \cap U_{l(i+1)}\right)$. Lift $\gamma_{1}=\gamma_{\mid\left[0, s_{1}\right]}$ to $\tilde{\gamma_{1}}$, with $\tilde{\gamma}_{1}(0)=y$, using the definable homoemorphism $p_{\mid V^{0}}: V^{0} \rightarrow U_{l(0)}$, where $V^{0}$ is the definable connected component of $p^{-1}\left(U_{l(0)}\right)$ in which $y$ lays. Repeat the process for each $\gamma_{i+1}=\gamma_{\mid\left[s_{i}, s_{i+1}\right]}$ with $\tilde{\gamma}_{i}\left(s_{i}\right)$ (intead of $y$ ). Patch the liftings together. Unicity follows (in each step) from Lemma 2.5.

Proposition 2.7 (Homotopy Lifting) Let $p: Y \rightarrow X$ be a definable covering map. Let $H:[a, b] \times[0,1] \rightarrow X$ be a definable homotopy between definable paths $\gamma(=H(a,-))$ and $\sigma(=H(b,-))$ and let $\tilde{\gamma}$ be a lifting of $\gamma$ to a definable path in $Y$. Then, there is a unique definable homotopy $\tilde{H}:[a, b] \times[0,1] \rightarrow Y$ between $\tilde{\gamma}$ and $\tilde{\sigma}$, lifting of $H$, where $\tilde{\sigma}$ is a lifting of $\sigma$ to a definable path in $Y$. 
Proof. Let $X=\bigcup_{l \in L} U_{l}$, as in the definition of definable covering map. Then $[a, b] \times[0,1]=\bigcup_{l \in L} H^{-1}\left(U_{l}\right)$, with the $H^{-1}\left(U_{l}\right)$ 's open in $[a, b] \times$ $[0,1]$. Then, by Lemma 6.3.6 in [6], we have that for each $l \in L$ there is a $W_{l} \subset[a, b] \times[0,1]$, open in $[a, b] \times[0,1]$ such that $W_{l} \subset \overline{W_{l}} \subset H^{-1}\left(U_{l}\right)$ and $[a, b] \times[0,1]=\bigcup_{l \in L} W_{l}$. Now decompose $\mathbf{M}^{2}$ compatible with the $W_{l}$ 's, this cell decomposition induce a cell decomposition $\left(a=t_{0}<t_{1}<\right.$ $\left.\cdots<t_{r}=b\right)$ of $[a, b]$. For each two-dimensional cell $C$ and each $s \in L$ such that $C \subset W_{s}$, we have $H(\bar{C}) \subset U_{s}$ and for any two-dimensional cells $C_{1}$ and $C_{2}$ in $[a, b] \times[0,1]$, and for each $s_{1}, s_{2} \in L$ such that $C_{1} \subset W_{s_{1}}$ and $C_{2} \subset W_{s_{2}}$ we also have $H\left(\overline{C_{1}} \cap \overline{C_{2}}\right) \subset U_{s_{1}} \cap U_{s_{2}}$. Now we proceed as above lifting each $H_{\mid \bar{C}}$ using the relevant definable homeomorphism and then patching the liftings together (as in the classical case but working with the two-dimensional cells instead of rectangles); we start with the closure of the bottom two-dimensional cell above $\left(t_{0}, t_{1}\right)$ and continue with the rest of the two-dimensional cells above $\left(t_{0}, t_{1}\right)$, patching the liftings together; then we consider the next column of two-dimensional cells above $\left(t_{1}, t_{2}\right)$ and we continue this way until we finish with the whole rectangle.

As above, unicity follows from the Unicity of Liftings (Lemma 2.5).

Note that if $H$ is a definable homotopy of paths all of them from $x$ to $x^{\prime}$ and $\tilde{\gamma}$ is a definable path from $y$ to $y^{\prime}$ then, by Unicity of Liftings Lemma 2.5, $\tilde{H}$ is a definable homotopy of paths all of them from $y$ to $y^{\prime}$. In particular if $H: \gamma_{1} \sim \gamma_{2}$ then $y * \gamma_{1}=y * \gamma_{2}$.

Having the above propositions at hand we can conclude, as in the classical case, with the following corollaries.

Corollary 2.8 Let $p: Y \rightarrow X$ be a definable covering map and let $p(y)=x$. Then, $p^{\pi}: \pi(Y, y) \rightarrow \pi(X, x)$ is an injective homomophism.

Proof. Since $p^{\pi}$ is a homomorphism, it suffices to prove that $p^{\pi}$ is injective. Let $[\sigma] \in \pi(Y)$ and suppose $[p \sigma]=\left[k_{x}\right]\left(k_{x}\right.$ constant path at $\left.x\right)$. Let $H: p \sigma \sim$ $k_{x}$ be a definable homotopy with $\gamma_{a}=p \sigma, \gamma_{b}=k_{x}$ and $x=\gamma_{s}(0)=\gamma_{s}(1)=$ $\gamma_{b}(t)$ for all $(s, t) \in[a, b] \times[0,1]$. By Homotopy Lifting Proposition 2.7, there is a unique definable homotopy $\tilde{H}$ of loops at $y, \tilde{H}: \sigma \sim \delta$. By unicity of the path lifting (comparing $\delta$ and $k_{y}$ ) we have $[\sigma]=\left[k_{y}\right]$.

Corollary 2.9 Let $p: Y \rightarrow X$ be a definable covering map and let $p(y)=x$. Then the following hold.

(a) Let $\sigma$ be a loop at $x$. Then, $y * \sigma=y$ if and only if $[\sigma] \in \operatorname{Im}\left(p^{\pi}\right)$.

(b) Let $\sigma$ and $\sigma^{\prime}$ be paths from $x$ to $x^{\prime}(\in X)$. Then, $y * \sigma=y * \sigma^{\prime}$ if and only if $\left[\sigma^{\prime} \cdot \sigma^{-1}\right] \in \operatorname{Im}\left(p^{\pi}\right)$. 
Proof. a) If $y * \sigma=y$, then $[\tilde{\sigma}] \in \pi(Y, y)$, and hence $[\sigma]=[p \tilde{\sigma}]=p^{\pi}[\tilde{\sigma}]$. For the converse suppose $[\sigma]=p^{\pi}\left[\sigma_{1}\right]\left(=\left[p \sigma_{1}\right]\right)$, and take $H: p \sigma_{1} \sim \sigma$ a definable homotopy of loops at $x$; by the Homotopy Lifting Proposition 2.7, $H$ lifts to a unique definable homotopy $\tilde{H}: \sigma_{1} \sim \gamma$ of loops at $y$ with $p \gamma=\sigma$, and hence $y * \sigma=\gamma(1)=y$.

b) Apply a) to the loop $\sigma^{\prime} \cdot \sigma^{-1}$ and use the unicity of path lifting of Proposition 2.6.

The next step is to adapt the concept of deck transformations to the definable context and to relate the corresponding group to the o-minimal fundamental group. Here again we have to pay attention to the definability condition, except for that the proofs are classical.

Let $p: Y \rightarrow X$ be a definable covering map. The group of definable covering transformations is Aut $_{p}^{\text {def }}(Y / X)=\{\varphi: Y \rightarrow Y \mid \varphi$ is a definable homeomorphism and $p \varphi=p\}$.

We shall omit the superscript "def" and the subscript " $p$ " if they are clear from the context.

Proposition 2.10 Let $p$ : $Y \rightarrow X$ be a definable covering map and let $p(y)=$ $x$. Suppose $p^{\pi}(\pi(Y, y)) \triangleleft \pi(X, x)$. Then, there is a canonical group isomorphism

$$
\pi(X, x) / p^{\pi}(\pi(Y, y)) \cong \operatorname{Aut}(Y / X) .
$$

Proof. We first define a group homomorphism $\psi: \pi(X) \rightarrow \operatorname{Aut}(Y / X)$, with $\psi([\sigma]): Y \rightarrow Y: w \mapsto \psi([\sigma])(w)=\varphi_{[\sigma]}(w)$, where $\varphi_{[\sigma]}$ is a definable covering transformation we proceed to define. Let $X=\bigcup_{l \in L} U_{l}$ and let $p^{-1}\left(U_{l}\right)=$ $\bigcup_{s \in S_{l}} V_{s}^{l}$, as in the definition of definable covering map, where the $V_{s}^{l}$ 's are the definably connected components of $p^{-1}\left(U_{l}\right)$. For each $l \in L$ and $s \in S_{l}$ (we shall omit the superscript $l$ ), choose $y_{s} \in V_{s}$, with $y_{s}=y$ if $y \in V_{s}$ and fix a definable path $\tau_{s}$ from $y$ to $y_{s}$. Now, for each $w \in V_{s}$, uniformily define a path $\delta_{s}(w)$ from $y_{s}$ to $w$ and let $\gamma_{s}(w)$ be $\tau_{s} \cdot \delta_{s}(w)$ which is a definable path from $y$ to $w$. Let $y^{\prime}=y * \sigma$ and define $\varphi_{[\sigma]}(w):=y^{\prime} *\left(p \circ \gamma_{s}(w)\right)(=$ $\left.y * \sigma \cdot\left(p \circ \gamma_{s}(w)\right)\right)$.

The rest of the proof follows from the definition and the previous established properties, as in the classical setting. Firstly, we check that the definition is independent of the definable path, from $y$ to $w$, chosen. Let $\gamma$ and $\gamma^{\prime}$ be two such paths. Then $\sigma \cdot(p \circ \gamma)$ and $\sigma \cdot\left(p \circ \gamma^{\prime}\right)$ are two definable paths from $x$ to $p(w)$, by Corollary 2.9 (b) $y * \sigma \cdot(p \circ \gamma)=y * \sigma \cdot\left(p \circ \gamma^{\prime}\right)$ if and 
only if $\left[\sigma \cdot\left(p \circ \gamma^{\prime}\right) \cdot(\sigma \cdot(p \circ \gamma))^{-1}\right] \in \operatorname{Im}\left(p^{\pi}\right)$. The class of the last loop is $[\sigma] p^{\pi}\left(\left[\gamma^{\prime} \cdot \gamma^{-1}\right]\right)[\sigma]^{-1}$, which belongs to the Image of $p^{\pi}$ by the normality assumption. Note also that by the Homotopy Lifting Proposition 2.7, the definition is also independent of any $\sigma^{\prime} \in[\sigma]$.

We now show that $\psi$ determines a left action of $\pi(X)$ on $Y: \pi(X) \times Y \rightarrow$ $Y:([\sigma], w) \mapsto[\sigma] \cdot w=\varphi_{[\sigma]}(w)$. Let $[\sigma],[\tau] \in \pi(X)$ and $w \in Y$. Let $\gamma$ be a definable path from $y$ to $w$, then the lifting of $\tau \cdot(p \circ \gamma)$ at $y$ is a definable path from $y$ to $[\tau] \cdot w$, and hence $[\sigma] \cdot([\tau] \cdot w)=y * \sigma \cdot(\tau \cdot(p \circ \gamma))$. On the other hand, the definable paths $(\sigma \cdot \tau) \cdot(p \circ \gamma)$ and $\sigma \cdot(\tau \cdot(p \circ \gamma))$ are definably homotopic and hence their lifts at $y$ have the same end points, by the Homotopy Lifting Proposition 2.7. The fact that $\left[k_{x}\right] \cdot w=w$ is easy.

Having the action, to prove that for each $[\sigma] \in \pi(X), \varphi_{[\sigma]} \in \operatorname{Aut}(Y / X)$, suffices to prove continuity of $\varphi_{[\sigma]}$. Let $w \in Y$ and fix $l \in L$ and $s \in S_{l}$ such that $p(w) \in U_{l}$ and $w \in V_{s}^{l}=V$. If $[\sigma] \cdot w \in V_{s}^{l^{\prime}}=V^{\prime}$, it suffices to prove that $[\sigma] \cdot V \subset V^{\prime}$. Let $v \in V$ and let $\alpha$ be a definable path from $w$ to $v$. Then $[\sigma] \cdot v=y^{\prime} * p \circ(\gamma \cdot \alpha)$, where $y^{\prime}=y * \sigma$, is the end point of a lifting of $p \circ \alpha$ at $[\sigma] \cdot v$.

To finish the proof we must show that $\psi$ is onto and it has the desired kernel. Let $\varphi \in \operatorname{Aut}(Y / X)$ with $\varphi(y)=y^{\prime}$. By the Unicity of Liftings Lemma 2.5, it suffices to find $[\sigma] \in \pi(X, x)$ such that $\varphi_{[\sigma]}(y)=y^{\prime}$. Let $\gamma$ be a definable path from $y$ to $y^{\prime}$ and let $\sigma$ be $p \circ \gamma$. Note that by definiton of definable covering transformation $\sigma$ is a definable loop at $x$, and check that this is the required $[\sigma] \in \pi(X)$. Finally, $\operatorname{Ker} \psi=\left\{[\sigma]: \psi([\sigma])=i d_{Y}\right\}=p^{\pi}(\pi(Y))$ by Corollary 2.9 (a).

We now apply the theory of definable covering maps to definable groups and obtain the following.

Proposition 2.11 Let $H$ and $G$ be definably connected definable groups. Let $h: H \rightarrow G$ be a definable onto homomorphism. Suppose ker $h$ is finite. Then, $h$ is a definable covering map and $\operatorname{ker} h \cong A_{h}(H / G)$.

Proof. Firstly, note that $h$ being a definable homomorphism between definable groups is continuous. The fact that $h$ is onto implies that there is a definable map $\alpha: G \rightarrow H$ such that $h \alpha=i d_{G}$. Since $\alpha$ is definable, there is a definable open subset $U$ of $G$ such that $\operatorname{dim}(G \backslash U)<\operatorname{dim} G$ and $\alpha_{\mid U}: U \rightarrow H$ is a definable continuous map. Let $V=h^{-1}(U)=\bigcup_{a \in k e r} V_{a}$, where $V_{a}=a \alpha(U)$. Note that for $a, b \in \operatorname{ker} h$, if $a \neq b$ then $V_{a} \cap V_{b}=\emptyset$, so we have have the open set $V$ as a disjoint finite union of definable open sets $V_{a}$ 's. Note also that $h_{\mid V_{a}}: V_{a} \rightarrow U$ is a definable homeomorphism for each $a \in k e r h$. 
On the other hand, since $U$ is large in $G$, by [11] Lemma 2.4, finitely many translates of $U$ cover $G, G=\bigcup_{t \in T} z_{t} U$, say. For each $t \in T$, take $y_{t} \in H$ with $h\left(y_{t}\right)=z_{t}$. Then for each $t \in T, h^{-1}\left(z_{t} U\right)=\bigcup_{a \in k e r h} y_{t} a \alpha(U)$, and the union is disjoint, moreover $h_{\mid y_{t} V_{a}}: y_{t} V_{a} \rightarrow z_{t} U$ is a definable homoemorphism. Now taking the definably connected components we obtain that $h$ is a definable covering map.

Having $h: H \rightarrow G$ a definable covering map and homomorphism of groups is easy to see that its kernel is isomorphic to $\operatorname{Aut}(H / G)$. Indeed, let $\psi: \operatorname{ker} h \rightarrow$ $\operatorname{Aut}(H / G)$ be defined as follows: for each $a \in \operatorname{ker} h, \psi(a): H \rightarrow H$ is left multiplicaton by $a$. Since $a$ is in the kernel of $h, \psi(a)$ is a definable covering transformation. Furthermore, $\psi$ is an injective homomorphism. We now show that $\psi$ is onto. Let $\varphi \in \operatorname{Aut}(H / G)$ and fix $z \in H$, then $a=\varphi(z) z^{-1}$ is in the kernel and $\varphi(z)=\psi(a)(z)$. By the Unicity Lemma $2.5 \varphi=\psi(a)$, as required.

Finally we are ready to prove Theorem 2.1.

Proof of Theorem 2.1. We consider, for each $k>0$, the map $p_{k}: G \rightarrow G: x \mapsto$ $k x$ (using additive notation) which is a definable homorphism. Firstly, note that definably connected abelian groups are divisible (and so the $p_{k}$ 's homorphisms are onto). Indeed, by Strzebonski [12], the torsion subgroups $G[k]=\operatorname{ker} p_{k}, k>0$ of $G$ are finite and hence $\operatorname{dim} G=\operatorname{dim} \operatorname{Im} p_{k}$, for each $k>0$; on the other hand $G$ is definably connected, so by [11], $G=\operatorname{dim} \operatorname{Im} p_{k}$. We can now apply Proposition 2.11 to conclude that each $p_{k}: G \rightarrow G$ is a definable covering map and that $G[k] \cong A u t_{p_{k}}(G / G)$. Furthermore, by Proposition 2.10 we obtain

$$
G[k] \cong \pi(G) /\left(p_{k}\right)^{\pi}(\pi(G)) .
$$

Now note that by Lemma 2.3, the finitely presented group $\pi(G)=\pi(G, 0)$ is abelian; and by Lemma 2.4 (using additive notation), we have $\left(p_{k}\right)^{\pi}(\pi(G))=$ $k \pi(G)$. To finish the proof it suffices to show that $\pi(G) \cong \mathbb{Z}^{s}$ for some $s \geq 0$. For this observe that the finitely generated abelian group $\pi(G)$ must be free abelian because, by Corollary 2.8, the map $\left(p_{k}\right)^{\pi}: \pi(G) \rightarrow \pi(G):[\sigma] \mapsto k[\sigma]$ is injective for each $k>0$.

Corollary 2.12 Let $G$ be a definably connected abelian group. Then, for each $k>0$, the homomorphism $p_{k}: G \rightarrow G: x \mapsto k x$ is a definable covering map. 


\section{O-minimal cohomology}

The aim of this section is to introduce the concept of o-minimal cohomology of a definable set and to prove that the o-minimal cohomology with coefficients in $\mathbb{Q}$ of a definably connected definable group can be equipped with a structure of Hopf-algebra which is isomorphic to a finitely generated exterior algebra over $\mathbb{Q}$.

Firstly, we consider the o-minimal homology theory developed by Woerheide in [13] (see also [1] and [7]). Given a definable set $X$ we consider, for each $m \geq 0$, the abelian group $S_{m}^{\text {def }}(X)$ freely generated by the singular definable simplexes $\sigma: \Delta_{m} \rightarrow X$, where $\Delta_{m}=\left\{\left(t_{0}, \ldots, t_{m}\right) \in \mathbf{M}^{m+1} \mid\right.$ $\left.\Sigma_{i} t_{i}=1, t_{i} \geq 0\right\}$ is the standard $m$-dimensional simplex. The boundary operator $\partial: S_{m+1}^{\text {def }}(X) \rightarrow S_{m}^{\text {def }}(X)$ (morphism of degree -1 ) is defined as in the classical case making $S_{*}^{\text {def }}(X)$ a free chain complex. Also, a definable continuous map $f: X \rightarrow Y$ induces a chain map $f_{\sharp}: S_{*}^{\text {def }}(X) \rightarrow S_{*}^{\text {def }}(Y)$ (i.e. a morphism of degree zero satifying $f_{\sharp} \partial=\partial f_{\sharp}$ ). Similarly one defines the definable singular chain complex of a pair of definable sets $Y \subset X$ : $S_{*}^{\text {def }}(X, Y)=S_{*}^{\text {def }}(X) / S_{*}^{\text {def }}(Y)$. The graded group $H_{*}^{\text {def }}(X)$ is defined as the homology of the chain complex $S_{*}^{\text {def }}(X)$. Similarly $H_{*}^{\text {def }}(X, Y)$ is the homology of $S_{*}^{\text {def }}(X, Y)$. A definable continuous map $f: X \rightarrow Y$ induces an homomorphism $f_{*}: H_{*}^{\text {def }}(X) \rightarrow H_{*}^{\text {def }}(X)$ of graded groups (via $f_{\#}$ ). The covariant functor $H_{*}^{\text {def }}$ satisfies the o-minimal Eilenberg-Steenrod homology axioms (analogues of the Eilenberg-Steenrod axioms for the category of definable sets and definable continuous maps). Note that if we consider, for each $m \geq 0$, the functor $S_{m}^{\text {def }}$ from the category of definable sets (and definable continuous maps) to the category of abelian groups, by definiton $S_{m}^{\text {def }}(X)$ is freely generated by $\left\{S_{m}^{\text {def }}(\sigma)\left(i d_{\Delta_{m}}\right) \mid \sigma: \Delta_{m} \rightarrow X\right.$, definable continuous $\}$, $\left(i d_{\Delta_{m}} \in S_{m}^{d e f}\left(\Delta_{m}\right)\right)$. This means that the functor $S_{m}^{d e f}$ is free with model the singleton $\left\{\Delta_{m}\right\}$ and basis $\left\{i d_{\Delta_{m}}\right\}$.

We shall omit the superscript "def" if it is clear from the context.

Lemma 3.1 Let $X$ be a definable set and let $\left\{X_{1}, \ldots, X_{s}\right\}$ be the definably connected components of $X$. Then,

(i) $H_{0}(X) \cong \mathbb{Z}^{s}$;

(ii) $H_{m}(X)=0$, for each $m \notin\{0, \ldots, \operatorname{dim} X\}$;

(iii) for each $m \geq 0, H_{m}(X) \cong \bigoplus_{j=1}^{s} H_{m}\left(X_{j}\right)$, and

(iv) for each $m \geq 0, H_{m}(X)$ is a finitely generated abelian group.

Proof. It can be easily obtained from the results in [13]. Otherwise, we can prove it by transfer, as follows. First suppose that $X$ is definably compact. 
So we can also assume $X=Y(\mathbf{M})$ (resp. $X_{i}=Y_{j}(\mathbf{M})(j=1, \ldots, s)$ the realization over $\mathbf{M}$ of some piecewise linear closed set $Y\left(\mathbb{Q}^{\text {ralg }}\right) \subset\left(\mathbb{Q}^{\text {ralg }}\right)^{l}$ $\left(\right.$ resp. $\left.Y_{j}(\mathbb{Q}) \subset\left(\mathbb{Q}^{\text {ralg }}\right)^{l}, j=1, \ldots, s\right)$, for some $l$, where $\mathbb{Q}^{\text {ralg }}$ are the real algebraic numbers. Then, by Proposition 3.2 in $[1], H_{m}^{\text {def }}(X) \cong H_{m}(Y(\mathbb{R}))$ and $H_{m}^{\text {def }}\left(X_{i}\right) \cong H_{m}\left(Y_{i}(\mathbb{R})\right.$ ), for each $m \geq 0$ and $j=1, \ldots, l$ (note that we are using the notation $H_{m}$ for the classical homology and $H_{m}^{\text {def }}$ for the o-minimal one). Then, also note that the lemma is true in the classical setting.

We reduce the general case to the definably compact case as in the proof of Theorem 1.1. in [1]. That is, by making use of a semialgebraic homeomorphism to a bounded set and a semialgebraic deformation retraction (this last one by Proposition 2.5 in [4])) to a definably compact set.

Consider the category $\mathcal{K}$ of pairs $(X, Y)$ of definable sets and pairs of definable continuous maps $(f, g):(X, Y) \rightarrow\left(X^{\prime}, Y^{\prime}\right)$. On $\mathcal{K}$ we define two functors to the category of chain complexes and chain maps: $S_{*}(X \times Y)$ and $S_{*}(X) \otimes S_{*}(Y)$. We have the following o-minimal version of the EilenbergZilber Theorem. (Compare with Dold [5] Theorem VI.12.1.)

Proposition 3.2 Let $X$ and $Y$ be definable sets. Then, there are unique (up to chain homotopy) natural chains maps

$$
\Psi: S_{*}(X \times Y) \leftrightarrows S_{*}(X) \otimes S_{*}(Y): \Phi
$$

such that $\Psi_{0}(\sigma, \tau)=\sigma \otimes \tau$ and $\Phi_{0}(\sigma \otimes \tau)=(\sigma, \tau)$ on definable singular 0-simplices $\sigma: \Delta_{0} \rightarrow X$ and $\tau: \Delta_{0} \rightarrow Y$. Any such chain map is a chain homotopy equivalence. In fact, each one of $\Phi \Psi$ and $\Psi \Phi$, is natural chain homotopic to the corresponding identity chain map. Any such chain map is call an EZ map.

Proof. The only difference with the classical proof is that we have to show in our definable category that $S_{*}(X \times Y)$ and $S_{*}(X) \otimes S_{*}(Y)$ are free and acyclic with models $\left\{\left(\Delta_{p}, \Delta_{q}\right)\right\}_{p, q \geq 0}$. With that aim we first consider the diagonal map $d_{m} \in S_{m}\left(\Delta_{m} \times \Delta_{m}\right)$. So that the singleton $\left\{d_{m}\right\}$ is a basis for $S_{m}(X \times Y)$, i.e., $S_{m}(X \times Y)$ is freely generated by $\left\{\left(\sigma_{1} \times\right.\right.$ $\left.\sigma_{2}\right)_{\#}\left(d_{m}\right) \mid\left(\sigma_{1}, \sigma_{2}\right):\left(\Delta_{m}, \Delta_{m}\right) \rightarrow(X, Y)$ definable continuous $\}$. Hence $S_{*}(X \times Y)$ is free with models $\left\{\left(\Delta_{m}, \Delta_{m}\right)\right\}_{m \geq 0}$ and a fortiori free with models $\left\{\left(\Delta_{p}, \Delta_{q}\right)\right\}_{p, q \geq 0}$. Now since $\Delta_{p}$ and $\Delta_{q}$ are definably contractible, so is $\Delta_{p} \times \Delta_{q}$ and hence the nonzero homology of $\Delta_{p} \times \Delta_{q}$ vanish. We have then proved that the functor $S_{*}(X \times Y)$ is a free acyclic functor with models $\left\{\left(\Delta_{p}, \Delta_{q}\right)\right\}_{p, q \geq 0}$. On the other hand, $S_{p}(X)$ (resp. $\left.S_{q}(X)\right)$ is free with basis 
the singleton $\left\{i d_{\Delta_{p}}\right\}$ (resp. $\left\{i d_{\Delta_{q}}\right\}$ ), hence $S_{p}(X) \otimes S_{q}(Y)$ is free with basis $\left\{i d_{\Delta_{p}} \otimes i d_{\Delta_{q}}\right\}$. Therefore, $S_{*}(X) \otimes S_{*}(Y)$ is free with models $\left\{\left(\Delta_{p}, \Delta_{q}\right)\right\}_{p, q \geq 0}$. Now the augmentation chain maps $\varepsilon: S_{*}(X) \rightarrow \mathbb{Z}$ and $\varepsilon: S_{*}(Y) \rightarrow \mathbb{Z}$ are chain equivalences and hence $\varepsilon \otimes \varepsilon: S_{*}(X) \otimes S_{*}(Y) \rightarrow \mathbb{Z} \otimes \mathbb{Z} \cong \mathbb{Z}$ is also a chain equivalence. Hence, the nonzero homology of $S_{*}(X) \otimes S_{*}(Y)$ vanish and we have shown that $S_{*}(X) \otimes S_{*}(Y)$ is also free and acyclic with models $\left\{\left(\Delta_{p}, \Delta_{q}\right)\right\}_{p, q \geq 0}$. Now apply the Acyclic Model Theorem to obtain the required result.

We observe that the $E Z$ maps are commutative, associative and preserve units (see [5] Theorem VI.12.1 and its Corollaries for details).

Now we consider two functors " $-\otimes \mathbb{Q}$ " and $" \operatorname{Hom}(-, \mathbb{Q})$ ", from the category of chain complexes of groups to the category of (co)chain complexes of $\mathbb{Q}$ vector spaces, in the usual way. Of course, we are interested in those chain complexes $S_{*}(X)$, where $X$ is a definable set, and chain maps $f_{\#}$, where $f: X \rightarrow Y$ is a definable continuous map. In this case, applying homology we obtain o-minimal homology with coefficients in $\mathbb{Q}$

$$
H_{m}(X ; \mathbb{Q})=H_{m}\left(S_{*}(X) \otimes \mathbb{Q}\right),
$$

and $f_{*}: H_{m}(X ; \mathbb{Q}) \rightarrow H_{m}(Y ; \mathbb{Q})$ induced by $f_{\#} \otimes i d$; and $o-m i n i m a l$ cohomology with coefficients in $\mathbb{Q}$

$$
H^{m}(X ; \mathbb{Q})=H_{-m}\left(\operatorname{Hom}\left(S_{*}(X), \mathbb{Q}\right)\right),
$$

and $f^{*}: H^{m}(Y ; \mathbb{Q}) \rightarrow H^{m}(X ; \mathbb{Q})$ induced by $\left.\operatorname{Hom}\left(f_{\#}, \mathbb{Q}\right)\right)$. These o-minimal homology and cohomology with coefficients in $\mathbb{Q}$ satisfy the corresponding Eilenberg-Steenrod axioms (see VI 7.1 in [5]). We can apply the Universal Coefficient Theorem and identify $H_{m}(X) \otimes \mathbb{Q}$ with $H_{m}(X ; \mathbb{Q})$ (and the corresponding $f_{*}$ 's), and $H^{m}(X ; \mathbb{Q})$ with $\operatorname{Hom}\left(H_{m}(X), \mathbb{Q}\right)$ (and $f^{*}$ with $\left.\operatorname{Hom}\left(f_{*}, \mathbb{Q}\right)\right)$ as $\mathbb{Q}$-vector spaces.

We observe that also the o-minimal (co)homology $\mathbb{Q}$-vector space is invariant under elementary extensions (see proofs of Proposition 3.2 in [1]).

By $\mathbb{Q}$-algebra we mean a commutative associative graded $\mathbb{Q}$-algebra with unit and all $\mathbb{Q}$-algebra morphism $h: \bigoplus_{m \geq 0} A_{m} \rightarrow \bigoplus_{m \geq 0} A_{m}^{\prime}$ are such that $h_{m}: A_{m} \rightarrow A_{m}^{\prime}$. We recall that $A$ is commutative if for $x \in A_{p}$ and $y \in$ $A_{q}, x \cdot y=(-1)^{p q} y \cdot x$. Next step is to equip the graded $\mathbb{Q}$-vector space $H^{*}(X ; \mathbb{Q})=\bigoplus_{m \geq 0} H^{m}(X ; \mathbb{Q})$ with a structure of $\mathbb{Q}$-algebra in a functorial way. With that aim we first prove an o-minimal Künneth Formula for cohomology as a corollary of the o-minimal Eilenberg-Zilber Theorem. 
Corollary 3.3 Let $X$ and $Y$ be definable sets. Then, there is a functorial isomorphism

$$
H^{*}(X \times Y ; \mathbb{Q}) \cong H^{*}(X ; \mathbb{Q}) \otimes H^{*}(Y ; \mathbb{Q}) .
$$

Proof. It is based on homological results except that at some point, we have to use the natural chain equivalence between $S_{*}(X \times Y)$ and $S_{*}(X) \otimes S_{*}(Y)$, obtained in Proposition 3.2 (see e.g., [5] Proposition VI.12.16). We give the steps of the proof (in [5]) for the convenience of the reader. Firstly, note that we can identify $\operatorname{Hom}\left(S_{*}(X), \mathbb{Q}\right)$ with $\operatorname{Hom}_{\mathbb{Q}}\left(S_{*}(X) \otimes \mathbb{Q}, \mathbb{Q}\right)$ for any definable set $X$, so that we can consider the four $\mathbb{Q}$-chain complexes $S_{*}(X) \otimes \mathbb{Q}, \mathbb{Q}, S_{*}(Y) \otimes \mathbb{Q}$ and $\mathbb{Q}$. On the other hand, $H_{*}(X ; \mathbb{Q})$ and $H_{*}(Y ; \mathbb{Q})$ are of finite type (actually, the are finite dimensional $\mathbb{Q}$-vector spaces, by Lemma 3.1 and the Universal Coefficient Theorem) and hence we have a natural chain homotopy equivalence $\operatorname{Hom}\left(S_{*}(X), \mathbb{Q}\right) \otimes_{\mathbb{Q}} \operatorname{Hom}\left(S_{*}(Y), \mathbb{Q}\right) \rightarrow$ $\operatorname{Hom}\left(S_{*}(X) \otimes S_{*}(Y), \mathbb{Q}\right)$ (see $\operatorname{VI}(10.23)$ in [5]). By the o-minimal EilenbergZilber Theorem, we can replace $S_{*}(X) \otimes S_{*}(Y)$ by $S_{*}(X \times Y)$ and still obtain a natural chain homotopy equivalence. Finally, we apply the Künneth Formula for chain complexes and obtain the required functorial isomorphism.

We can now define a product in $H^{*}(X ; \mathbb{Q})$, for $X$ a definable set. Let $d: X \rightarrow X \times X: x \mapsto(x, x)$ be the diagonal map. Identifying $H^{*}(X \times X ; \mathbb{Q})$ with $H^{*}(X ; \mathbb{Q}) \otimes H^{*}(X ; \mathbb{Q})$, through the o-minimal Künneth Formula for cohomology (Corollary 3.3), we obtain

$$
d^{*}: H^{*}(X ; \mathbb{Q}) \otimes H^{*}(X ; \mathbb{Q}) \rightarrow H^{*}(X ; \mathbb{Q}): x \otimes y \mapsto d^{*}(x \otimes y):=x \cdot y,
$$

where $x \in H^{p}(X ; \mathbb{Q})$ and $y \in H^{q}(X ; \mathbb{Q})$. The properties of the EZ map (see Proposition 3.2) imply that the product - is associative, commutative in the graded sense and has a unit $1 \in H^{0}(X ; \mathbb{Q})$. Naturality over the whole procedure makes also the product structure on $H^{*}(X ; \mathbb{Q})$ functorial. Therefore, we have proved the following.

Theorem 3.4 Let $X$ be e definable set. Then, the cohomology graded $\mathbb{Q}$ vector space $H^{*}(X ; \mathbb{Q})$ can be equipped with a structure of $\mathbb{Q}$-algebra. Furthermore, if $Y$ is a definable set, any definable continuous map $f: X \rightarrow Y$ induces a $\mathbb{Q}$-algebra morphism $f^{*}: H^{*}(Y ; \mathbb{Q}) \rightarrow H^{*}(X ; \mathbb{Q})$.

A $\mathbb{Q}$-algebra $A=\bigoplus_{m \geq 0} A_{m}$ is a connected $\mathbb{Q}$-algebra if $A_{0} \cong \mathbb{Q}$; we shall denote the unit of $A$ (image of $1 \in \mathbb{Q}$ ) by 1 , and $\bigoplus_{m>0} A_{m}$ by $D^{1}(A)$. A comultiplication in a connected $\mathbb{Q}$-algebra is a morphism $\mu: A \rightarrow A \otimes A$, such 
that for any $x \in D^{1}(A), \mu(x)=x \otimes 1+1 \otimes x+r$, with $r \in D^{1}(A) \otimes D^{1}(A)$. A connected $\mathbb{Q}$-algebra $A$ equipped with an associative comultiplication $\mu$ is called a Hopf-algebra over $\mathbb{Q}$.

Corollary 3.5 Let $G$ be a definably connected definable group and let $m$ : $G \times$ $G \rightarrow G$ denote the multiplication in $G$. Then $H^{*}(G ; \mathbb{Q})$ can be equipped with a structure of Hopf-algebra over $\mathbb{Q}$, with comultiplication $\mu: H^{*}(G ; \mathbb{Q}) \rightarrow$ $H^{*}(G ; \mathbb{Q}) \otimes H^{*}(G ; \mathbb{Q})$ induced by $m$.

Proof. Firstly, we apply Theorem 3.4 to give $H^{*}(G ; \mathbb{Q})$ a structure of $\mathbb{Q}$ algebra. Now, since $G$ is definably connected $H_{0}(G) \cong \mathbb{Z}$, and so $H^{0}(G ; \mathbb{Q}) \cong$ $\mathbb{Q}$ and hence $H^{*}(G ; \mathbb{Q})$ is a connected $\mathbb{Q}$-algebra. Next we identify the o-minimal cohomology $H^{*}(G \times G ; \mathbb{Q})$ with $H^{*}(G ; \mathbb{Q}) \otimes H^{*}(G ; \mathbb{Q})$ via the Künneth Formula. Hence, Theorem 3.4 again gives us a $\mathbb{Q}$-algebra morphism $\mu=m^{*}: H^{*}(G ; \mathbb{Q}) \rightarrow H^{*}(G ; \mathbb{Q}) \otimes H^{*}(G ; \mathbb{Q})$. Now checking that $\mu$ is a comultiplication is standard. Indeed, let $x \in D^{1}\left(H^{*}(G ; \mathbb{Q})\right)$ and suppose $\mu(x)=y \otimes 1+1 \otimes z+r$ with $r \in D^{1}\left(H^{*}(G ; \mathbb{Q})\right) \otimes D^{1}\left(H^{*}(G ; \mathbb{Q})\right)$, we have to show that $y=z=x$. Let $e$ denote the neutral element of $G$ and $i:\{e\} \rightarrow G$ the inclusion map. Then the composition $G \times\{e\} \stackrel{i d_{G} \times i}{\longrightarrow} G \times G \stackrel{m}{\longrightarrow} G$ takes $(a, e)$ to $a$, for each $a \in G$. Then, identifying $H^{*}(G \times\{e\} ; \mathbb{Q})$ with $H^{*}(G ; \mathbb{Q}) \otimes \mathbb{Q}$ (and $H^{*}(G \times G ; \mathbb{Q})$ with $\left.H^{*}(G ; \mathbb{Q}) \otimes H^{*}(G ; \mathbb{Q})\right)$ via Künneth, we can consider that the map $\left(i d_{G} \times i\right)^{*}: H^{*}(G ; \mathbb{Q}) \otimes H^{*}(G ; \mathbb{Q}) \rightarrow H^{*}(G ; \mathbb{Q}) \otimes \mathbb{Q}$ takes $u \otimes w \in H^{p}(G ; \mathbb{Q}) \otimes H^{q}(G ; \mathbb{Q})$ to $u \otimes w$ if $u \in H^{0}(G ; \mathbb{Q})$ and to 0 otherwise, therefore, $x \otimes 1=y \otimes 1(\neq 0)$. Similarly we obtain $x=z$ via the map $i \times i d_{G}$.

Corollary 3.6 Let $G$ be a definably connected group and let $m$ denote the multiplication on $G$. Then the Hopf-algebra $H^{*}(G ; \mathbb{Q})$, with comultiplication $m^{*}$ is isomorphic to the exterior algebra $\bigwedge\left[y_{1}, \ldots, y_{r}\right]_{\mathbb{Q}}$, with the $y_{i}$ 's of odd degree and primitive, i.e., $m^{*}\left(y_{i}\right)=y_{i} \otimes 1+1 \otimes y_{i}$, for each $i=1, \ldots, r$.

Proof. We are over a field of zero characteristic, so the Hopf-Leray Theorem says that our Hopf-algebra $H^{*}(G ; \mathbb{Q})$ is a free (commutative) $\mathbb{Q}$-algebra. Moreover, it can be generated as a $\mathbb{Q}$-algebra by a set $P$ of primitive (or indecomposable) elements (see e.g., [5] VII.10.16 or Brown [3] III.F). Since $H^{j}(G ; \mathbb{Q})=0$ for all $j>\operatorname{dim} G$, and $H^{j}(G ; \mathbb{Q})$ is a finite dimensional $\mathbb{Q}$ vector space (by Lemma 3.1 and the Universal Coefficient Theorem), the set $P$ must be finite. For the same reason, $P$ cannot contain elements of even degree, since $x$ of even degree implies $x^{l} \neq 0$ for all $l \in \mathbb{N}$. Therefore, $H^{*}(G ; \mathbb{Q}) \cong \bigwedge\left[y_{1}, \ldots, y_{r}\right]_{\mathbb{Q}}$ 
Note that, as a $\mathbb{Q}$-vector space, each $H^{j}(G ; \mathbb{Q})$ is generated by 1 if $j=0$, and by all monomials $y_{i_{1}} \cdots \cdots y_{i_{s}}$ with $1 \leq i_{1}<\cdots<i_{l} \leq r$ and $\sum_{s=1}^{l} \operatorname{deg}\left(y_{i_{s}}\right)=j$ if $j>0$. So, if all $y_{i}$ 's are of degree one, the dimension of $H^{j}(G ; \mathbb{Q})$ is $\left(\begin{array}{l}r \\ j\end{array}\right)$.

\section{Degree of a definable map}

In this section we introduce the notion of degree of a definable map (under some suitable hypothesis) and we establish its basic properties. The aim is to prove that for $G$ as in the hypothesis of the Structure Theorem 1.1 the degree of the map $p_{k}: G \rightarrow G: x \rightarrow k x$, with $k>0$, is bounded above by $\left|p_{k}^{-1}(0)\right|(=|G[k]|)$. This is intuitively obvious if we think of the degree of a (definable covering) map as the number of preimages counted with their multiplicities. We need some preliminaries.

A definable orientation in a definable manifold $X$ of dimension $n$ is a map $s$ which assigns to each point $p \in X$ a generator $s(p)$ of the local homology group $H_{n}^{\text {def }}(X, X \backslash p)$ which is locally constant in the following sense: for each point in $X$ there is a definably compact neighbourhood (of the point) $N$ and a class $\zeta_{N} \in H_{n}^{\text {def }}(X, X \backslash N)$ such that for each $p \in N$ the natural homomorphism $H_{n}^{\text {def }}(X, X \backslash N) \rightarrow H_{n}^{\text {def }}(X, X \backslash p)$, induced by the inclusion map $(X, X \backslash N) \rightarrow(X, X \backslash p)$, sends $\zeta_{N}$ into $s(p)$ (see [2]). Given the following data: an oriented definable manifold $X$ of dimension $n$, a definable orientation $s$ in $X$, and a definably compact definable set $N \subset X$. Then, by the proof of Theorem 5.2 in [2], there is a unique relative homology class $\zeta_{N} \in H_{n}^{\text {def }}(X, X \backslash N)$, the fundamental class around $N$, such that for each $p \in N$ the homomorphism $H_{n}^{\operatorname{def}}(X, X \backslash N) \rightarrow H_{n}^{\operatorname{def}}(X, X \backslash p)$, induced by the inclusion map $i^{p}:(X, X \backslash N) \rightarrow(X, X \backslash p)$, sends $\zeta_{N}$ into $s(p)$ (note that the group $H_{n}^{\text {def }}(X, X \backslash N)$ does not need to be cyclic). If $U$ is an open definable subset of $X$ containing $N$ we shall identify $\zeta_{N}$ with its image through the excision isomorphism $H_{n}^{\text {def }}(X, X \backslash N) \stackrel{\cong}{\rightrightarrows} H_{n}^{\text {def }}(U, U \backslash N)$. Note that if $N=\emptyset$ then $\zeta_{N}=0$ and that if $N_{1} \subset N_{2}$ are definably compact definable subsets of $X$, then $\zeta_{N_{1}}$ is the image of $\zeta_{N_{2}}$ through the homomorphism induced by the inclusion $i:\left(X, X \backslash N_{2}\right) \rightarrow\left(X, X \backslash N_{1}\right)$. If $X$ is definably compact we call $\zeta_{X}$ the fundamental class of $X$ for the given orientation.

We shall omit the superscript "def" if it is clear from the context

Proposition 4.1 Let $X$ be a definably compact oriented definable manifold of dimension $n$. Let $N$ be a nonempty definably connected definably compact subset of $X$. Then $H_{n}(X, X \backslash N) \cong \mathbb{Z}$, and $\zeta_{N}$ is a generator of $H_{n}(X, X \backslash$ 
$N)$. Moreover, for any open definable set $U$ with $N \subset U \subset X$, we have $H_{n}(U, U \backslash N) \cong \mathbb{Z}$.

Proof. By transfer. We can suppose, as in the proof of Lemma 3.1, that $X=Y(\mathbf{M})$ and $N=A(\mathbf{M})$ are the realizations over $\mathbf{M}$ of some piecewise linear sets $Y\left(\mathbb{Q}^{\text {ralg }}\right)$ and $A\left(\mathbb{Q}^{\text {ralg }}\right)$, with $Y\left(\mathbb{Q}^{\text {ralg }}\right)$ closed. Since $Y(\mathbf{M})$ is a definable manifold, by Lemmas 4.10 and 4.11 in $[1], Y(\mathbb{R})$ is a homology manifold. On the other hand, the existence of a fundamental class around $A(\mathbf{M})$ makes the group $H_{n}^{\text {def }}(Y(\mathbf{M}), Y(\mathbf{M}) \backslash A(\mathbf{M}))$ nontrivial. Then, by transfer of homology (see [2] §2), the relative homology group $H_{n}(Y(\mathbb{R}), Y(\mathbb{R}) \backslash A(\mathbb{R}))(\cong$ $\left.H_{n}^{\text {def }}(Y(\mathbf{M}), Y(\mathbf{M}) \backslash A(\mathbf{M}))\right)$ is not trivial. Since $(Y(\mathbb{R}), Y(\mathbb{R}) \backslash A(\mathbb{R}))$ is a compact triangulated relative homology manifold of dimension $n$ with $A(\mathbb{R})$ connected, we have $H_{n}(Y(\mathbb{R}), Y(\mathbb{R}) \backslash A(\mathbb{R})) \cong \mathbb{Z}$ (see Munkres [8], Corollary 70.4). Again by transfer of homology, we obtain $H_{n}^{\text {def }}(X, X \backslash N) \cong \mathbb{Z}$ (Note that we are using the notation $H_{n}$ for the classical homology and $H_{n}^{\text {def }}$ for the o-minimal one). The last statement is obtained by excision.

We are now in situation of adapting the classical notion of degree of a map to the o-minimal context. We follow [5] noting that by Propositon 4.1, we need the further hypothesis: $X$ is definably compact, to get $H_{n}(X, X \backslash N)$ cyclic.

Let $f: Y \rightarrow X$ be a definable continuous map between oriented definable manifolds of dimension $n$, with $X$ definably compact. Let $N$ be a definably compact definably connected nonempty definable subset of $X$. Suppose $f^{-1}(N)$ is definably compact. Consider the image of the fundamental class around $f^{-1}(N)$, through the map $f_{*}: H_{n}\left(Y, Y \backslash f^{-1}(N)\right) \rightarrow H_{n}(X, X \backslash N)$. By Proposition 4.1, this map takes $\zeta_{f^{-1}(N)}$ into an integral multiple of $\zeta_{N}$, this integer is called the degree of $f$ over $N$ and it is denoted by $\operatorname{deg}_{N} f$, i.e., $f_{*}\left(\zeta_{f^{-1}(N)}\right)=\operatorname{deg}_{N} f \zeta_{N}$; if moreover $Y$ is definably compact and $X$ is definably connected then $\operatorname{deg} f:=\operatorname{deg}_{X} f$ is called the degree of $f$. Note that $\operatorname{deg}_{N} f=0$ if $f^{-1}(N)=\emptyset$.

The next lemmas establish some basic properties of the degree. Their proofs are as in the clasical case, and as usual we include them for completeness.

Lemma 4.2 Let $Y$ and $X$ be oriented definable manifolds of dimension $n$ with $X$ definably compact. Let $V$ be a definable open subset of $Y$. Then the following holds.

(a) Let $f: V \rightarrow X$ be the inclusion map (between oriented manifolds). Let $N$ be a nonempty definably connected definably compact definable subset of $V$. Then, $\operatorname{deg}_{N} f=1$. 
(b) Let $f: Y \rightarrow X$ be a definable homeomorphism onto and open subset of $Y$. Suppose $N$ is a nonempty definably connected definably compact definable subset of $V$ such that $f^{-1}(N)$ is definably compact. Then, $\operatorname{deg}_{N} f= \pm 1$.

Proof. (a): The excision isomorphism $H_{n}(X, X \backslash N) \stackrel{e x c}{\cong} H_{n}(V, V \backslash X)$ is the inverse of $f_{*}: H_{n}(V, V \backslash N) \rightarrow H_{n}(X, X \backslash N)$ and hence $f_{*}\left(\zeta_{N}\right)=\zeta_{N}$ (we have identified $\zeta_{N}$ with its image through the excision isomorphism).

(b): Since $H_{n}(X, X \backslash N)$ is cyclic, by excision $H_{n}(f(Y), f(Y) \backslash N)$ is also cyclic. Therefore, the composition $H_{n}\left(Y, Y \backslash f^{-1}(N)\right) \stackrel{f_{*}}{\cong} H_{n}(f(Y), f(Y) \backslash$ $N) \stackrel{i_{*}}{\cong} H_{n}(X, X \backslash N)$ must take $\zeta_{f^{-1}(N)}$ to $\pm \zeta_{N}$.

Lemma 4.3 Let $f: Y \rightarrow X$ be a definable continuous map between oriented definable manifolds of dimension $n$, with $X$ definably compact. Let $N \subset N_{1}$ be definably compact nonempty subsets of $X$ such that $N$ is definably connected and $f^{-1}(N)$ and $f^{-1}\left(N_{1}\right)$ are definably compact. Then, $f_{*}: H_{n}\left(Y, Y \backslash f^{-1}\left(N_{1}\right)\right) \rightarrow H_{n}\left(X, X \backslash N_{1}\right)$ takes $\zeta_{f^{-1}\left(N_{1}\right)}$ into $\left(\operatorname{deg}_{N} f\right) \zeta_{N_{1}}$; if moreover $N_{1}$ is definably connected then $\operatorname{deg}_{N} f=\operatorname{deg}_{N_{1}} f$.

Proof. Consider the following commutative diagram

$$
\begin{aligned}
& H_{n}\left(Y, Y \backslash f^{-1}(N)\right) \stackrel{f_{*}}{\rightarrow} \quad H_{n}(X, X \backslash N) \\
& H_{n}\left(Y, Y \backslash f^{-1}\left(N_{1}\right)\right) \stackrel{f_{*}}{\rightarrow} H_{n}\left(X, X \backslash N_{1}\right),
\end{aligned}
$$

where $i_{*}$ and $j_{*}$ are induced by the respective inclusion maps. Chasing $\zeta_{f^{-1}(N)}$ through the diagram gives $\zeta_{f^{-1}(N)} \mapsto \operatorname{deg}_{N} f \zeta_{N} \mapsto \operatorname{deg}_{N} f \zeta_{N_{1}}$, respectively $\zeta_{f^{-1}(N)} \mapsto \zeta_{f^{-1}\left(N_{1}\right)} \mapsto f_{*}\left(\zeta_{f^{-1}\left(N_{1}\right)}\right)$

Corollary 4.4 Let $f: Y \rightarrow X$ be a definable continuous map between definably compact oriented definable manifolds of dimension $n$ such that $X$ is definably connected.Then $\operatorname{deg}_{q} f=\operatorname{deg} f$, for any $q \in X$.

Lemma 4.5 Let $f: Y \rightarrow X$ be a definable continuous map between oriented definable manifolds of the same dimension such that $X$ is definably compact. Let $N \subset X$ be a definably compact set such that $f^{-1}(N)$ is definably compact. Suppose $Y=\bigcup_{\lambda=1}^{m} Y_{\lambda}$ such that each $Y_{\lambda}$ is an open definable subset of $Y$ and $f^{-1}(N)$ is the disjoint union of the $f^{-1}(N) \cap Y_{\lambda}$ 's. Then $\operatorname{deg}_{N} f=$ $\sum_{\lambda=1}^{m} \operatorname{deg}_{N} f^{\lambda}$, where $f^{\lambda}=f_{\mid Y_{\lambda}}: Y_{\lambda} \rightarrow X$. 
Proof. Firstly, note that the $N_{\lambda}^{\prime}=f^{-1}(N) \cap Y_{\lambda}$ are clopen in $f^{-1}(N)$ and hence definably compact, so it makes sense to speak about the fundamental class around $N_{\lambda}^{\prime}$. Then observe that if $i^{\lambda}$ is the inclusion map $\left(Y_{\lambda}, Y_{\lambda} \backslash N_{\lambda}^{\prime}\right) \rightarrow$ $\left(Y, Y \backslash f^{-1}(N)\right)$ the the following diagram commutes.

$$
\begin{array}{ccc}
\oplus_{\lambda=1}^{m} H_{n}\left(Y_{\lambda}, Y_{\lambda} \backslash N_{\lambda}^{\prime}\right) \stackrel{\left(i_{*}^{\lambda}\right)}{\longrightarrow} & H_{n}\left(Y, Y \backslash f^{-1}(N)\right) \\
\sum_{\lambda=1}^{m} f_{*}^{\lambda} \searrow & \downarrow f_{*} \\
& H_{n}(X ; X \backslash N) .
\end{array}
$$

Now, for each $p \in f^{-1}(N)$, consider the maps $\oplus_{\lambda=1}^{m} H_{n}\left(Y_{\lambda}, Y_{\lambda} \backslash N_{\lambda}^{\prime}\right) \stackrel{\left(i_{*}^{\lambda}\right)}{\rightarrow}$ $H_{n}\left(Y, Y \backslash f^{-1}(N)\right) \stackrel{i_{*}^{p}}{\rightarrow} H_{n}(Y, Y \backslash p)$ and observe that $i_{*}^{p}\left(\left(i_{*}^{\lambda}\right)\left(\zeta_{N_{\lambda}^{\prime}}\right)\right)=s(p)$ (all the components of $\left(\zeta_{N_{\lambda}^{\prime}}\right)$ go to zero except the component $\zeta_{N_{\mu}^{\prime}}$ which goes to $s(p))$. Hence, by unicity of the fundamental class $\left(i_{*}^{\lambda}\right)\left(\zeta_{N_{\lambda}^{\prime}}\right)=\zeta_{f^{-1}(N)}$. Therefore, by the above diagram, we have $\left(\operatorname{deg}_{N} f\right) \zeta_{N}=f_{*}\left(\zeta_{f^{-1}(N)}\right)=$ $f_{*}\left(\left(i_{*}^{\lambda}\right)\left(\zeta_{N_{\lambda}^{\prime}}\right)\right)=\sum_{\lambda=1}^{m} f_{*}^{\lambda}\left(\zeta_{N_{\lambda}^{\prime}}\right)=\left(\sum_{\lambda=1}^{m} \operatorname{deg}_{N} f^{\lambda}\right) \zeta_{N}$.

We already know that a definable group carries a structure of definable manifold. The fact that $G$ is orientable is classical: choose a local orientation around 0 say, and extend it to the whole group using the operation of the group (see proof of Corollary 3.4 in [2]). Finally we can prove the following.

Proposition 4.6 Let $G$ be a definably compact definably connected abelian definable group. For each $k>0$, consider the map $p_{k}: G \rightarrow G: x \mapsto k x$. Then, $G$ is an orientable definable manifold and for any orientation on $G$, we have deg $p_{k} \leq\left|p_{k}^{-1}(0)\right|$, where 0 is the neutral element of $G$.

Proof. Fix an orientation on $G$ and a $k>0$. By Corollary 4.4, deg $p_{k}=$ $\operatorname{deg}_{0} p_{k}$. Also, by Corollary 2.12, we know that the homomophism $p_{k}: G \rightarrow G$ is a definable covering map. Let $G=\bigcup_{l \in L} U_{l}$ be as in the definition of covering map. Fix $l_{o} \in L$ such that $0 \in U_{l_{o}}$ and let $Y=p_{k}^{-1}\left(U_{l_{o}}\right)$. Now consider the map $f=\left(p_{k}\right)_{\mid Y}: Y \rightarrow G$. Note that, by excision, $\operatorname{deg}_{0} p_{k}=$ $\operatorname{deg}_{0} f$. On the other hand, if we let $Y=\bigcup_{\lambda=1}^{m} Y_{\lambda}$ with the $Y_{\lambda}$ 's being the definably connected components of $Y$ so that $f^{\lambda}=f_{\mid Y_{\lambda}}: Y \rightarrow G$ is a homeomorphism onto an open subset of $G$, namely $U_{l_{o}}$. Now the data $f: Y \rightarrow G,\{0\} \subset G$ and $Y=\bigcup_{\lambda=1}^{m} Y_{\lambda}$ satisfy the hypothesis of Lemma 4.5, therefore we can conclude that $\operatorname{deg}_{0} f=\sum_{\lambda=1}^{m} \operatorname{deg}_{0} f^{\lambda}$. Finally, by Lemma $4.2, \operatorname{deg}_{0} f^{\lambda}= \pm 1$, for each $\lambda$, and hence $\operatorname{deg}_{0} f \leq m=\left|f^{-1}(0)\right|$. By the above, this last relation means $\operatorname{deg} p_{k} \leq\left|p_{k}^{-1}(0)\right|$. 


\section{Proof of the Structure Theorem}

We first prove an o-minimal version of the Poincaré-Hurewitz Theorem. For a group $G$, let $G_{a b e l}$ denote the abelianization of $G$, i.e. the quotient of $G$ by its commutator subgroup.

Theorem 5.1 Let $X$ be a definably connected set. Then, the abelianization of the o-minimal fundamental $\pi(X)_{\text {abel }}$ of $X$ is isomorphic to the first ominimal homology group $H_{1}(X)$ of $X$.

Proof. By transfer. As in the proof of Lemma 3.1 we can assume that $X$ is definably compact and that $X=Y(\mathbf{M})$, the realization over $\mathbf{M}$ of some piecewise linear closed set $Y\left(\mathbb{Q}^{\text {ralg }}\right) \subset\left(\mathbb{Q}^{\text {ralg }}\right)^{l}$ (see proof of Lemma 3.1). Then, by Proposition 3.2 and Theorem 1.1 in $[1], H_{1}^{\text {def }}(X) \cong H_{1}(Y(\mathbb{R}))$ and $\pi^{\operatorname{def}}(X) \cong \pi(Y(\mathbb{R}))$. Therefore, by the classical Poincaré-Hurewitz Theorem, $H_{1}^{\text {def }}(X) \cong H_{1}(Y(\mathbb{R})) \cong \pi(Y(\mathbb{R}))_{a b e l} \cong \pi^{\text {def }}(X)_{a b e l}$.

Consider now a definably connected group $G$ (not necessary abelian). Our next goal is to compute, for each $k>0$, the Hopf-algebra morphism $p_{k}^{*}$ induced by the definable continuous map $p_{k}: G \rightarrow G: a \mapsto a^{k}$, for each $a \in G$. We have all the ingredients to follow exactly the computations in [3] III.F. By Corollary 3.6, we know that there are elements $y_{1}, \ldots, y_{r} \in H^{*}(G ; \mathbb{Q})$ such that $H^{*}(G ; \mathbb{Q})$ is generated, as a graded $\mathbb{Q}$-vector space, by $1 \in H^{0}(G ; \mathbb{Q})$ and all the monomials $y_{i_{1}} \cdots y_{i_{l}}$, where $1 \leq i_{1}<\cdots<i_{l} \leq r$; we say that such a monomial has length $l$ and write len for length. Moreover, if $m$ is the multiplication of $\mathrm{G}$, then $m^{*}: H^{*}(G ; \mathbb{Q}) \otimes H^{*}(G ; \mathbb{Q}) \rightarrow H^{*}(G ; \mathbb{Q})$, takes $y$ to $m^{*}(y)=y \otimes 1+1 \otimes y$ for each $y \in\left\{y_{1}, \ldots, y_{r}\right\}$.

Lemma 5.2 Let $G$ be a definably connected group. For each $k>0$, consider the definable continuous map $p_{k}: G \rightarrow G: a \rightarrow a^{k}$, for each $a \in G$. Then, the map $p_{k}^{*}: H^{*}(G ; \mathbb{Q}) \rightarrow H^{*}(G ; \mathbb{Q})$ sends each monomial $x$ to $k^{\text {len } x} x$.

Proof. Firstly, for $y$ a primitive element of positive degree, which is one of the generators of $H^{*}(G ; \mathbb{Q})$ as a Hopf-algebra (i.e., $\left.y \in\left\{y_{1}, \ldots, y r\right\}\right)$, we prove that $p_{k}^{*}(y)=k y$, by induction on $k$. Note that, for $k=1, p_{k}$ is the identity on $G$, and $p_{k+1}=m \circ\left(p_{k} \times i d\right) \circ d$, where $d: G \rightarrow G \times G$ is the diagonal map in $G$. Also note that if $f, g: G \rightarrow G$ are definble continuous maps we can assume, through the Kunneth identification, that $(f \times g)^{*}=f^{*} \otimes g^{*}$ Then, $p_{k+1}^{*}(y)=\left(m \circ\left(p_{k} \times i d\right) \circ d\right)^{*}(y)=\left(d^{*} \circ\left(p_{k} \times i d_{G}\right)^{*} \circ m^{*}\right)(y)=$ $d^{*} \circ\left(p_{k}^{*} \otimes i d\right)(y \otimes 1+1 \otimes y)=d^{*}(k y \otimes 1+1 \otimes y)=k y \cdot 1+1 \cdot y=(k+1) y$. Finally, we get $p_{k+1}^{*}(x)=(k+1)^{\text {len } x} x$, for each $k>0$, since $p_{k+1}^{*}$ is an algebra morphism. 
Proof of the Structure Theorem 1.1:

Let $G$ be a definably connected definably compact abelian group of dimension $n$. Let $H^{*}(G ; \mathbb{Q})=\bigwedge\left[y_{1}, \ldots, y_{r}\right]_{\mathbb{Q}}$ as in Corollary 3.6. Fix an orientation on $G$ and let $\zeta_{G} \in H_{n}(G)$ be the fundamental class of $G$ for the given orientation (see the proof of Corollary 4.6). Since $G$ is definably connected and definably compact we know, by Proposition 4.1, that $\zeta_{G}$ is a generator of the cyclic group $H_{n}(G)$. Let $\omega_{G}$ be the corresponding cohomology class in $H^{n}(G ; \mathbb{Q})$, so that $\omega_{G}\left(\zeta_{G}\right)=1$. Now fix a $k>0$, and consider the definable continuous map $p_{k}: G \rightarrow G: a \rightarrow k a$. By definition of degree of a map we obtain $p_{k}^{*}\left(\omega_{G}\right)=\left(\operatorname{deg} p_{k}\right) \omega_{G}$. Since $\omega_{G}$ generates $H^{n}(G ; \mathbb{Q})$, and $0 \neq y_{1} \cdots y_{r} \in H^{n}(G ; \mathbb{Q})$ we can suppose $\omega_{G}=y_{1} \cdots y_{r}$. By Lemma 5.2, $p_{k}^{*}\left(\omega_{G}\right)=k^{r} \omega_{G}$, and so $\operatorname{deg} p_{k}=k^{r}$.

On the other hand, by Theorem 2.1 , there is an $s \geq 0$ such that $\pi(G) \cong \mathbb{Z}^{s}$ and $\left(p_{k}\right)^{-1}(0)=G[k] \cong(\mathbb{Z} / k \mathbb{Z})^{s}$. By Corollary 4.6, deg $p_{k} \leq\left|\left(p_{k}\right)^{-1}(0)\right|=$ $k^{s}$, and hence $r \leq s$. By Theorem 5.1, $\left(\mathbb{Z}^{s} \cong\right) \pi(G) \cong H_{1}(G)$, and hence $\mathbb{Q}^{s} \cong H^{1}(G ; \mathbb{Q})$. Since $H^{1}(G ; \mathbb{Q})$ is a subspace of $H^{*}(G ; \mathbb{Q})$ (and the elements of $H^{1}(G ; \mathbb{Q})$ cannot be decomposable), among $\left\{y_{1}, \ldots, y_{r}\right\}$ must be exactly $s$ of degree one. Hence $s=r$ and all $y_{i}$ 's must be of degree one. Finally, observe that $\omega_{G}=y_{1} \cdots y_{r} \in H^{n}(G ; \mathbb{Q})$ to conclude that $s=r=n$.

\section{References}

[1] A. Berarducci and M. Otero, O-minimal fundamental group, homology and manifolds, J. London Math. Soc. 65 (2002) 257-270

[2] A. Berarducci and M. Otero, Transfer methods for o-minimal topology, J. Symbolic Logic 68 (2003) 785-794

[3] R. Brown, The Lefschetz Fixed Point Theorem, Scott, Foresman and Company 1971

[4] H. Delfs and M. Knebusch, On the homology of algebraic varieties over real closed fields, J. reine u. angew. Math 335 (1982) 122-163

[5] A. Dold, Lectures on Algebraic Topology, second ed., Springer 1980

[6] L. van den Dries, Tame Topology and o-minimal structures, LMS 248, Cambridge Univ. Press 1998

[7] M. Edmundo and A. Woerheide, Comparation theorems for o-minimal singular (co)homology, RAAG Preprint n.54 (http://ihp-raag.org/), April 2004, 39pp. 
[8] J. Munkres, Elements of Algebraic Topology, Addison Wesley 1984

[9] Y.Peterzil and S.Starchenko, Definable homomorphisms of abelian groups definable in o-minimal structures, Ann. Pure Appl. Logic 101 (1999) 1-27

[10] Y. Peterzil and C. Steinhorn, Definable compacteness and definable subgroups of o-minimal groups, J. London Math. Soc. 59 (1999) 769-786

[11] A.Pillay, On groups and fields definable in o-minimal structures, J. Pure Appl. Algebra 53 (1988) 239-255

[12] A. Strzebonski, Euler characteristic in semialgebraic and other $o^{-}$ minimal structures, J. Pure Appl. Algebra 96 (1994) 173-201

[13] A. Woerheide, O-minimal homology, $\mathrm{PhD}$ Thesis, University of Illinois at Urbana-Champaign 1996 